of two to four months). At $\$ 190$ the journal represents good value, and it is pleasing to see that is moving from three to four issues in the forthcoming year. Overall, JEPP would certainly be a worthwhile addition to library stocks for institutions with specific research or teaching interests in environmental policy.

i) http://www.rsc.org/is/journals/current/jem/jempub.htm http://www.interscience.wiley.com/jpages/1523-908X Graham Wood is in the Impacts Assessment Unit, School of Planning, Oxford Brookes University, Oxford OX3 OBP, UK.

\section{More than a breath of fresh air}

\section{Wind Energy}

editor-in-chief Robert W. Thresher

Wiley. 4/yr. \$320 (institutional),

$\$ 245$ (individual)

\section{Ian Fells}

The wind bloweth where it listeth; and thou hearest the sound thereof, butcanst not tell whence it cometh and whither it goeth.

St John, ch. 3, v. 8

Man has harnessed the wind to power sailing latterly, to generate electricity. Wind is fickle and unpredictable, as those who race yachts may know to their cost. But now wind energy, or rather, electricity generated by huge wind turbines - each producing two megawatts of power - is big business and growing at 30\% a year. Worldwide there are 15 gigawatts of wind-generated electricity capacity.

As is so often the case, the practical engineering is well ahead of the science. The international journal Wind Energy has been set up to try to redress this imbalance and "offers a major forum for the reporting of advances in this rapidly developing technology ... to ships, turn windmills to grind corn and,

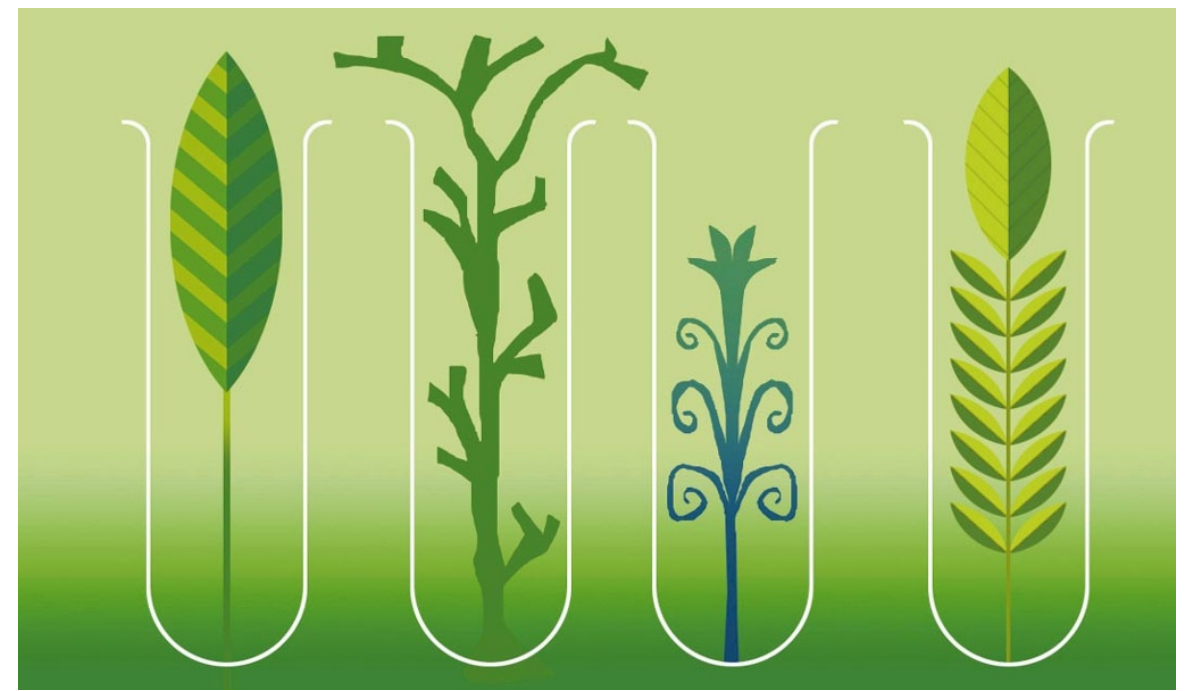

harness clean energy from the wind".

The papers range widely, from "Wind power meteorology", through "Modelling methods for wind turbine wakes" to "Mature offshore wind technology". They are generally rigorous, with a nice balance of theoretical and practical content. The fluid mechanics of wind systems is crucial for energy to be generated efficiently and the journal's contents reflect this. Surprisingly, despite the journal's mission statement, the economics of wind-generated electricity and sociopolitical issues seem not to feature as yet, although a section on "Broader perspectives" is promised.

There is some competition from Wind Directions, a journal published by the European Wind Energy Association, and from Renewable Energy World, published by Edward Milford, but these are essentially trade magazines and so Wind Energy fills an important gap.

\section{http://wwww3.interscience.wiley.com/cgi-bin/jtoc?ID}

\section{$=6276$}

Ian Fells is principal consultant at Fells Associates, 29 Rectory Terrace, Newcastle upon Tyne NE3 1YB, UK.
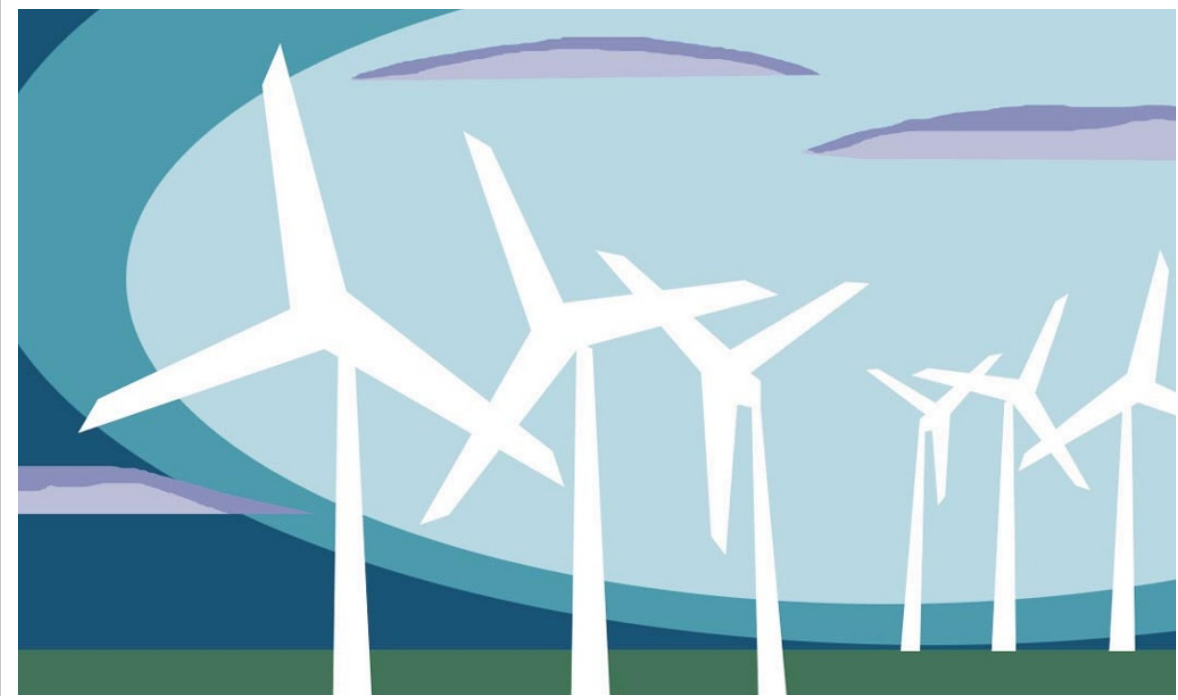

\section{Chemists exploring a verdant field}

\section{Green Chemistry}

editorial board chairman Roger A. Sheldon Royal Society of Chemistry. 6/yr. £250, \$412 (institutional, including site-wide electronic access), £45, \$72 (individual)

\section{Walter Leitner}

"Green chemistry" may sound like an oxymoron to those who identify chemistry with cheap and nasty industry, and associate "green" with fundamental activists fighting against technological progress. But the need for clean and environmentally benign processes based on a sustainable development is now widely recognized and has found its way into many corporate targets and research policies. In this context, "green chemistry" is defined as the utilization of a set of principles that reduces or eliminates the use or generation of hazardous substances in the design, manufacture and application of chemical products (P. Anastas \& J. C. Warner, Green Chemistry, Oxford Univ. Press, 1998).

Despite its seemingly clear focus on technological application, the above definition results in a tremendous challenge for fundamental science in chemistry. What impact do chemicals have on the environment? How can we develop alternatives to potentially hazardous reagents? Is it possible to replace traditional reaction sequences resulting in the inevitable generation of by-products with new transformations where all starting materials are incorporated in the product? Can we design, or efficiently screen for, new catalysts that make reactions cleaner or help to save energy? What are the chances of reaction media? These are just some of the questions that organic and inorganic synthesis, physical chemistry and chemical engineering can help to address in this field. replacing organic solvents with more benign 
The new journal Green Chemistry provides an international forum for discussion and scientific exchange of this fairly heterogeneous community, which is held together by a common goal rather than a specific methodology or a specialized subject.

Each of the six annual issues contains two sections - News \& Views and Research Publications. The first, slightly shorter, part highlights new developments in technology, research policy or science and provides information about symposia, funding programmes and ongoing projects. In the second part, about ten scientific papers deal mainly with the topics described above and include experimental procedures, usually in detail. The quality of layout and graphics is excellent throughout the journal, making it easy to scan the contents if time is short. The reasonably priced institutional subscription includes site-wide electronic access, and additional personal subscriptions to the printed version are available at very moderate rates.

Owing to its rather broad definition, the field of green chemistry is still in flux: in this situation, the publication of a periodical plays an important role in delineating the exact scope of a field. At the moment, each research article in Green Chemistry is accompanied by a box in which one of the editors describes the "green context" of the work. The journal is well on the way towards making the need for this explanation obsolete, and has the potential to establish its position in the chemical literature.

\section{http://www.rsc.org/is/journals/current/green/}

\section{greenpub.htm}

Walter Leitner is at the Max-Planck-Institut für

Kohlenforschung, Kaiser-Wilhelm-Platz 1,

D-45470 Mülheim an der Ruhr, Germany.

\section{.}

\section{Chemistry's voice}

\section{heard at last}

\section{Foundations of Chemistry: Philosophical, Historical, Educational and Interdisciplinary Studies of Chemistry \\ editor-in-chief Eric R. Scerri \\ Kluwer. 3/yr. \$124 (institutional), \\ $\$ 70$ (individual)}

\section{Bernadette Bensaude-Vincent}

Twentieth-century philosophy of science especially the anglophone tradition — virtually ignored chemistry. From logical positivism through to Popper, Kuhn, Lakatos and Feyerabend, physics has been the model science. This neglect of the philosophy of chemistry prompted a strong reaction in the 1990s in Britain, Germany and the United States. After a number of conferences and symposia in 1994, two journals were created to fill the gap: Hyle: An International Journal for the Philosophy of Chemistry in 1995, published by the University of Karlsruhe in Germany, and Foundations of Chemistry in 1999.

If we admit that 'filling a gap' is more than a rhetorical justification for new journals, the main question is, 'how do you fill the gap?'. The answer is not clear from the four issues of Foundations of Chemistry. Its editor-in-chief Eric Scerri, an American chemist, turned to the philosophy of chemistry in order to fight against the reduction of chemistry to quantum physics. His initial motivation was to demonstrate that the foundations of chemistry lie in chemistry itself. For him, the philosophy of chemistry is mainly a strategy to claim chemistry's autonomy.

The journal's title clearly indicates that it is a response of the chemical community to the longstanding neglect of chemistry which began with Foundations of the Unity of Science, the famous publication of the logical positivism movement. Scerri, aware of the dangers of a reactive attitude, is trying to broaden the scope of the journal to include such topics as the role of instruments in chemistry, and to develop a historical dimension lacking in logical positivism. However, the success of the enterprise will rely on the journal's ability to interest philosophers and historians of science. Its future depends on the formation of a multidisciplinary and multicultural research community of philosophers of chemistry.

\section{http://www.wkap.nl/journalhome.htm/ \\ 1386-4238}

Bernadette Bensaude-Vincent is in the Département de Philosophie, Université Paris X-Nanterre, 92001 Nanterre Cedex, France.

\section{Struggling with complexity}

\section{Crystal Engineering}

editor-in-chief Mike Zaworotko

Pergamon-Elsevier. 4/yr.

euro $166.54, \$ 186$

\section{Gastone Gilli}

Reductionism has been very successful when applied in a descending order of scale and complexity, the normal direction for reducing biology to chemistry, solidstate physics and, finally, elementaryparticle physics. Unfortunately, as remarked in 1972 by physics nobellist Philip Anderson, "the ability to reduce everything to simple fundamental laws does not imply the ability to start from those laws and

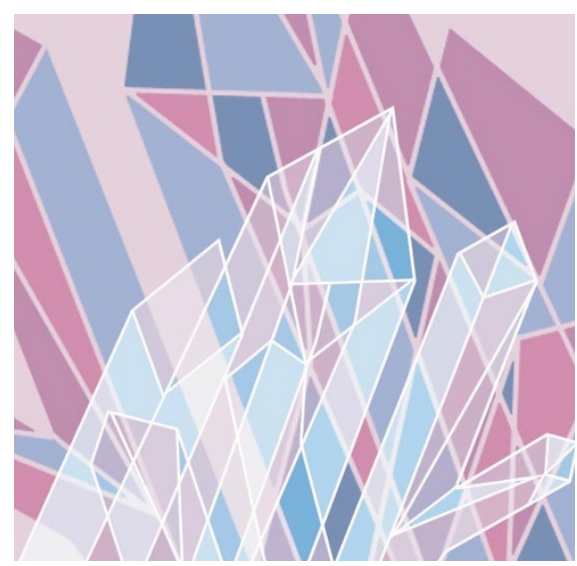

reconstruct the universe ... Instead, at each level of complexity, entirely new properties appear." This makes it next to impossible to deduce the complexity and novelty that can emerge through knowing the composition of many elementary entities.

Anderson's views are regularly verified in the new cross-disciplinary field of crystal engineering. The related journal is aimed at developing protocols for predicting and controlling the structure and function of molecular crystalline materials. Although the basic laws governing molecular interactions at the sub-microscopic level are essentially known, the preferred forms of molecular aggregation at the upper level of complexity (the macroscopic crystal) remain substantially unpredictable.

This may justify the journal's editorial policy, which focuses on the synthesis and determination of the structure of molecular crystals, co-crystals and clathrates that have specific and potentially useful internal patterns. There is less emphasis on theoretical interpretation or ab initio crystal structure prediction. The journal therefore adds to the harvest of experimental data from which the final rules of crystal composition will, hopefully, emerge.

Crystal Engineering has other merits, in particular a pleasing typographic form and a truly outstanding list of editorial members. Its success will depend on its ability to complement other well-established crystallographic journals by becoming a reference for general problems of crystal design, such as the systematics of molecular interactions, the topochemical features of crystals that give them their specific physical properties, and the rationalization of the often cryptic supramolecular nomenclature.

\section{http://www.crystal-engineering.net}

Gastone Gilli is in the Department of Chemistry, Centre for Structural Diffractometry,

University of Ferrara, Via Borsari 46, I-44100 Ferrara, Italy. 